\title{
Cocrystallization phenomenon of polyoxymethylene blend samples between the deuterated and hydrogenated species
}

\author{
Thontree Kongkhlang ${ }^{1}$, Kummetha Raghunatha Reddy ${ }^{1}$, Toshiaki Kitano ${ }^{2}$, Takashi Nishu ${ }^{2}$ and Kohji Tashiro ${ }^{1}$ \\ The phenomenon of cocrystallization between the deuterated $(D)$ and hydrogenated $(H)$ species of a series of polyoxymethylene \\ blends with various $H / D$ ratios has been established on the basis of the detailed analysis of thermal and infrared (IR) spectral \\ data. The melting and crystallization temperatures were found to shift continuously toward a higher temperature with \\ an increase in the D content. The IR spectra were found to change remarkably depending on the H/D blend content. Both the \\ thermal and the IR spectral observations definitely indicated the cocrystallization between the $H$ and $D$ chains in the common \\ crystallite. These continuous changes could be interpreted by assuming that the arrays of the $H$ and $D$ chains in crystal lattice \\ are random.
}

Polymer Journal (2011) 43, 66-73; doi:10.1038/pj.2010.106; published online 17 November 2010

Keywords: cocrystallization; deuterated species; DSC; infrared spectra; isotopic blend; polyoxymethylene

\section{INTRODUCTION}

Semicrystalline polymers show complex microstructures or higherorder structures that consist of crystalline and amorphous regions. Control of the higher-order structure may govern the physical properties of the polymers. A better understanding of the formation process is required in order to control the higher-order structure of the polymer and develop new materials with better properties. As the first step, the aggregation state of the molecular chains in the crystallized bulk sample must be determined at the molecular level. As the polymer solid is built up by the complicated aggregation of more or less entangled chains, it is difficult to trace the trajectory of a single particular chain in the bulk sample. The isotopic blend between the deuterated (D) and hydrogenated $(\mathrm{H})$ species is considered one of the most useful methods for studying the behavior of a single chain in the aggregation state on the basis of the difference in the thermal and vibrational characters that are due to the difference in atomic mass. ${ }^{1-22}$ In some cases, the difference in the neutron scattering length is also utilized successfully to distinguish the $\mathrm{D}$ and $\mathrm{H}$ species. ${ }^{14,17,18}$

In such cases of isotopic blends, the cocrystallization must be confirmed to take place between the $\mathrm{H}$ and $\mathrm{D}$ species in the crystalline lattice. For example, the blends between the $\mathrm{H}$ and $\mathrm{D}$ species of the various types of polyethylene have been comprehensively studied for clarification of the cocrystallization phenomenon between the $\mathrm{H}$ and D species. ${ }^{1-4,8-11,14,16-19}$ In the case of samples that are a blend of $\mathrm{H}$ high-density polyethylene and $\mathrm{D}$ high-density polyethylene, slow cooling from the melting point, in which the $\mathrm{D}$ (or $\mathrm{H}$ ) chains gathered together in the $\mathrm{H}$ (or D) matrix, showed the phase segregation phenomenon. ${ }^{2}$ However, the cocrystallization phenomenon was observed for the blend samples between $\mathrm{D}$ high-density polyethylene and $\mathrm{H}$ linear low-density polyethylene with about 17 ethyl branches/ 1000 carbon atoms even when cooled slowly from the melting point. ${ }^{9-11,16-19}$ The $\mathrm{D} / \mathrm{H}$ blend samples that show this perfect cocrystallization phenomenon are useful for the tracing of a single chain during the crystallization process and also for the clarification of the chain folding mode in the crystallization phenomenon. In fact, the systematic interpretation of the various kinds of data obtained from the blend samples of D high-density polyethylene/linear low-density polyethylene (17 ethyl branching) lead to a constructive conclusion concerning the aggregation state of the chains and chain folding in the crystalline lamellae. ${ }^{18}$ A similar study has been made for the $\mathrm{D} / \mathrm{H}$ blend samples of isotactic polypropylene, where the helical chains of the $\mathrm{D}$ and $\mathrm{H}$ species coexist randomly in the common crystal lattice. ${ }^{20}$

In the present paper, polyoxymethylene (POM, $\left.\left[-\mathrm{CH}_{2} \mathrm{O}-\right]\right)$ is treated as another typical example of the cocrystallization of the $\mathrm{D}$ and $\mathrm{H}$ species, when cooled from the melting point. POM is one of the most important engineering thermoplastics: it is commonly used to replace metals and alloys because of its high tensile strength, impact resistance, stiffness, good dimensional stability and corrosion resistance. In the crystal structure of POM, the non-uniform (9/5) helical chains are packed in the trigonal unit cell of $a=4.47 \AA$ and

${ }^{1}$ Department of Future Industry-oriented Basic Science and Materials, Graduate School of Engineering, Toyota Technological Institute, Tempaku, Nagoya, Japan and ${ }^{2}$ Department of Applied Chemistry, Graduate School of Engineering, Nagoya University, Chikusa-ku, Nagoya, Japan

Correspondence: Dr K Tashiro, Department of Future Industry-oriented Basic Science and Materials, Graduate School of Engineering, Toyota Technological Institute, Tempaku, Nagoya 468-8511, Japan.

E-mail: ktashiro@toyota-ti.ac.jp

Received 20 July 2010; revised 10 September 2010; accepted 16 September 2010; published online 17 November 2010 
$c=17.39 \AA^{23}$ It should be noted that, the crystal structure is more complicated, as described by Tashiro et al. ${ }^{24}$ in their analysis of synchrotron X-ray and neutron diffraction data. The morphological structure of the crystalline phase may be classified into two types as extreme cases, an extended-chain crystal (ECC) and a folded chain crystal (FCC) or lamellar crystal, depending on the crystallization condition as well as the thermal and mechanical histories. ${ }^{25-28}$ The physical properties of POM are significantly influenced by such crystal morphologies. The typical FCC structure is achieved by crystallization from a dilute solution, which generates hexagon-shaped lamellar crystals with a thickness of about $10 \mathrm{~nm}$ where the molecular chains are folded back on the lamellar surfaces, with the stems aligning perpendicular to the surface. ${ }^{29}$ The typical ECC structure is achieved through the heterogeneous cationic polymerization of trioxane, resulting in micron-sized needle-like single crystals, in which the extended POM molecules align parallel to the needle axis. ${ }^{30,31}$ The crystal morphology found in the melt-grown bulk samples lies between the two above mentioned extremes. ${ }^{32-34}$ The ECC type is also developed on the drawing of the sample. ${ }^{28}$

Among the various techniques available for the investigation of the morphology of a polymer, Fourier transform infrared spectroscopy (FTIR) may be one of the most powerful methods for POM because of its uniqueness and high sensitivity to changes in the morphology, as shown by Shimomura et al. ${ }^{26}$ The ECC and FCC structures give the remarkably different FTIR spectra, although they show essentially the same X-ray diffraction pattern. ${ }^{26}$ The IR bands with transition dipole moments parallel to the chain axis (parallel bands; $A_{2}$ ) show a high frequency shift (as high as $100 \mathrm{~cm}^{-1}$ ) for the FCC sample compared with the ECC sample, whereas the IR bands with transition dipole moments perpendicular to the chain axis (perpendicular bands; $E_{1}$ ) are unaltered. The drastic shift of the parallel bands depending on the morphology can be interpreted by assuming that there are long-range interactions among the transition dipoles. ${ }^{27,28}$

The blending of the $\mathrm{H}$ and $\mathrm{D}$ species of POM may affect the IR spectral profile sensitively. This will result in more complicated IR spectra as both the effect of the morphology and the isotope species must be taken into account. Kobayashi et al. ${ }^{25}$ reported IR spectral changes for a sample that was a blend between normal and D paraformaldehydes (PFA and PFA- $d_{2}$, linear oligomers of POM). The $A_{2}$ parallel bands exhibited remarkable changes in both the peak position and the intensity with variation in the PFA/PFA- $d_{2}$ composition. However, cocrystallization between the $\mathrm{H}$ and $\mathrm{D}$ chains has not yet been experimentally established for these blend samples. To clarify the isotope effect on the FTIR spectral changes and to disclose the aggregation state of the molecular chains in the crystallized bulk sample, the cocrystallization between the $\mathrm{H}$ and $\mathrm{D}$ chains must be confirmed experimentally. The present work therefore aims to clarify the cocrystallization behavior between the $\mathrm{H}$ and D species of POM utilizing thermal and vibrational spectroscopic techniques.

\section{EXPERIMENTAL PROCEDURE}

\section{Samples}

We used the hydrogenated polyoxymethylene (POM-H) Duracon M90 with $M_{\mathrm{w}}$ ca. $69300 \mathrm{~g} \mathrm{~mol}^{-1}$ and $M_{\mathrm{w}} / M_{\mathrm{n}}$ ca. 2.3, which was supplied by Polyplastics, Shizuoka, Japan. Duracon M90 is a copolymer of trioxane with a small amount of ethylene oxide, about $2.2 \mathrm{wt} \%$, which is added to avoid thermal decomposition. The deuterated polyoxymethylene (POM-D, $M_{\mathrm{w}} \mathrm{ca} .140000 \mathrm{~g} \mathrm{~mol}^{-1}, M_{\mathrm{w}} / M_{\mathrm{n}}$ ca. 2.3) was synthesized by a solid-state polymerization reaction of $\mathrm{D}$ trioxane single crystal under $\gamma$-ray irradiation $\left({ }^{60} \mathrm{Co}, 6 \mathrm{kGy}\right.$ at $\left.60{ }^{\circ} \mathrm{C}\right) .{ }^{35,36} 1,1,1,3,3,3$ Hexafluoro-2-propanol (bp $58^{\circ} \mathrm{C}$ ) was used as a solvent. The samples that were cooled from the melt had to be treated carefully. As revealed in previous papers, ${ }^{32}$ the POM samples were heated above the melting points of both the $\mathrm{H}$ and the $\mathrm{D}$ species and maintained for $5 \mathrm{~min}$. A longer duration of the molten state caused thermal degradation, and a shorter duration was insufficient to melt the crystallite completely.

POM-H/D blended samples of 100:0, 90:10, 70:30, 50:50, 30:70, 10:90 and 0:100 molar ratios were prepared by dissolving POM-H and POM-D in 1,1,1,3,3,3-hexafluoro-2-propanol at an ambient temperature, followed by precipitation into acetone with vigorous stirring. The precipitated samples were dried in a vacuum at $80^{\circ} \mathrm{C}$. The samples have been denoted here as POM- $\mathrm{H}^{* *}$, with ${ }^{* *}$ corresponding to the $\mathrm{mol} \%$ of the $\mathrm{H}$ species used (for example, POM-H50 (50 mol\% $\mathrm{H}$ species and $50 \mathrm{~mol} \% \mathrm{D}$ species $)$ ).

\section{Measurements}

The thermograms were measured by a TA instrument differential scanning calorimeter (DSC) Q1000 under nitrogen gas flow at a heating rate of $2{ }^{\circ} \mathrm{Cmin}^{-1}$ from 25 to $200^{\circ} \mathrm{C}$. The IR spectra were measured using an FT7000 Varian rapid-scan-type FTIR spectrometer at a resolution power of $2 \mathrm{~cm}^{-1}$. The X-ray diffraction profile was measured using a Rigaku/TTR-III $\mathrm{X}$-ray diffractometer with a $\mathrm{Cu}-\mathrm{K} \alpha$ line as an incident X-ray beam in the $2 \theta$ range of $10-60^{\circ}$ at a scanning rate $5^{\circ} \mathrm{Cmin}^{-1}$. The crystallite size along the chain axis $(l)$ was estimated using Scherrer's equation, $l=K \lambda /(\beta \cos \theta)$, where $K$ is a constant (0.9), $\lambda$ is the wavelength of the incident X-ray beam (1.542 $\AA$ ), $\beta$ is a full width at a half maximum peak intensity (FWHM) and $2 \theta$ is a Bragg angle. The FWHM was corrected for the peak broadening effect caused by the slit system of the diffractometer, in which $\beta^{2}=\beta_{\mathrm{obs}}^{2}-\beta_{\mathrm{m}}^{2}$, where $\beta_{\mathrm{obs}}$ was the observed FWHM and $\beta_{\mathrm{m}}$ was the FWHM observed for the Si powder sample.

\section{Isothermal crystallization}

The samples were heated above the melting temperature and kept at that temperature for $5 \mathrm{~min}$ before quenching to the predetermined crystallization temperature $\left(T_{\mathrm{c}}\right)$ at a cooling rate of $70^{\circ} \mathrm{C} \mathrm{min}^{-1}$. An optimal melting condition had been reported by Hama et al. ${ }^{32}$ to erase any remaining effect of the residual crystalline lamellae and to avoid sample decomposition. We followed the procedures described by them. The temperature was kept at $T_{\mathrm{c}}$ until crystallization was completed. The crystallized samples were then quenched to the ambient temperature at a rate of $70^{\circ} \mathrm{C} \mathrm{min}^{-1}$ for evaluation of the crystallite thickness $(l)$. The evaluation of the melting temperature $T_{\mathrm{m}}(l)$ of these samples was made by reheating at $2{ }^{\circ} \mathrm{C} \mathrm{min}^{-1}$.

\section{RESULTS AND DISCUSSION}

\section{Cocrystallization phenomena as viewed by thermal analysis}

Figure 1 shows the DSC thermograms that were measured for the blend samples POM-H100, POM-H50 and POM-H0. The single melting $\left(T_{\mathrm{m}}\right)$ and crystallization $\left(T_{\mathrm{c}}\right)$ peaks were observed to occur in the blend sample at a temperature that was between the melting temperatures of the original pure $\mathrm{H}$ and $\mathrm{D}$ samples. The inset pattern shown in Figure $1 \mathrm{~b}$ indicates a second $T_{\mathrm{c}}$, which might be related to the formation of the ECC structure of POM or the straight tie chains passing through the neighboring lamellae. ${ }^{32}$ It should be noted that the endothermic peak from the melting of ECC was particularly difficult to detect in the heating process of the H/D blend sample. The DSC peak is generally broader during the heating process than it is during the cooling process; this difference can be typically seen in the main melting and crystallization peaks, as shown in this figure. A similar situation may apply to the ECC melting peak as well. The H/ $\mathrm{D}$ content dependence of the DSC thermograms that were measured during the heating and cooling processes is shown in Figure 2. The $T_{\mathrm{m}}$ and $T_{\mathrm{c}}$ were found to shift continuously toward a higher temperature with an increase in the D content, implying that cocrystallization was occurring between the $\mathrm{H}$ and $\mathrm{D}$ chains. It should be pointed out that cocrystallization can occur even when the sample is cooled slowly from the melting point $\left(2{ }^{\circ} \mathrm{Cmin}^{-1}\right)$. Figure 3 shows the $T_{\mathrm{m}}$ and $T_{\mathrm{c}}$ plotted against the H/D content, clearly demonstrating the above mentioned situation. Figure 4 shows a continuous shift of the second 

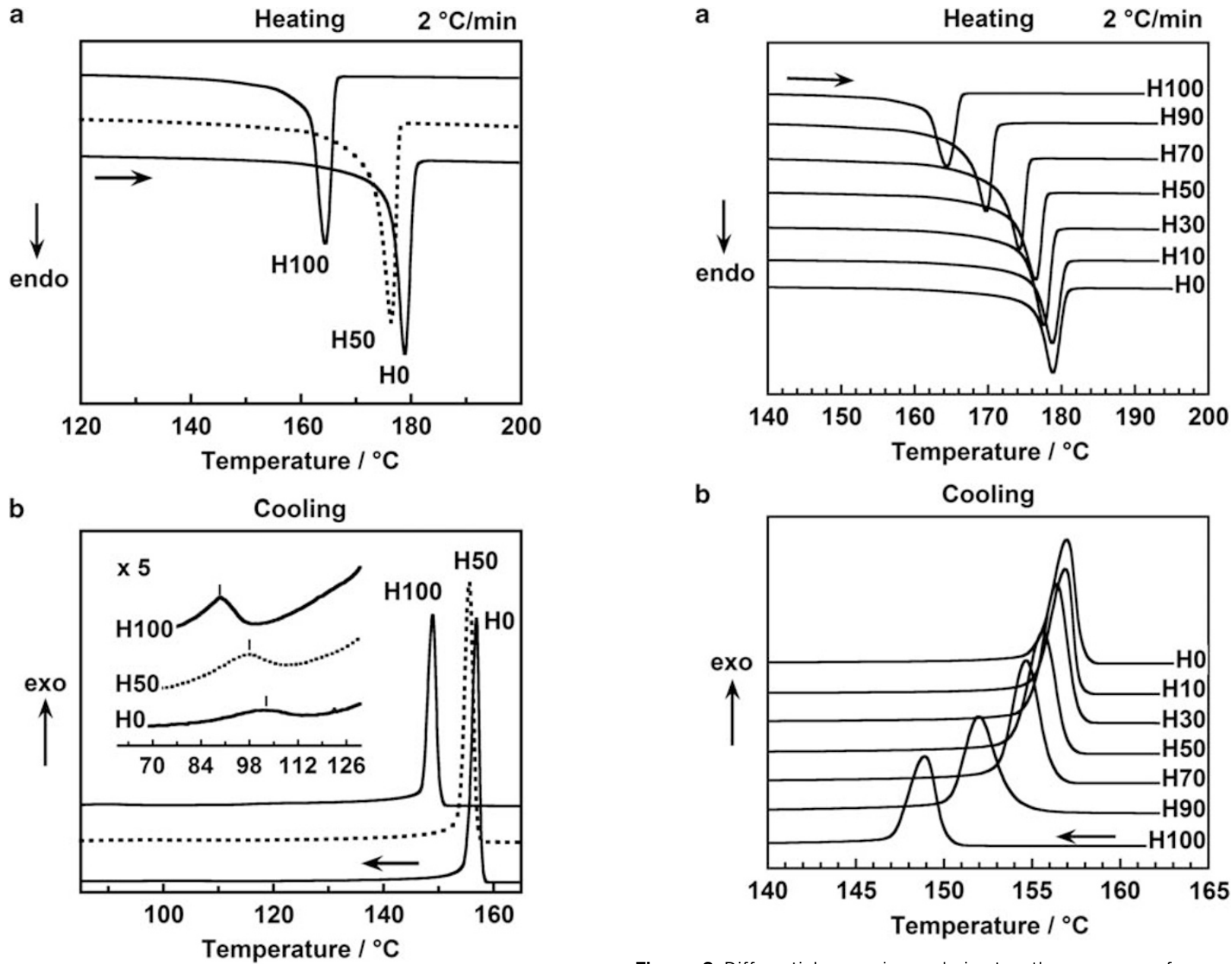

Figure 1 Differential scanning calorimeter thermograms of the polyoxymethylene (POM)-H100, - $\mathrm{H} 50$ and - $\mathrm{H} 0$ samples measured during (a) the heating and (b) the cooling processes, with an inset indicating the second crystallization temperature $T_{\mathrm{c} 2} . \mathrm{H}$, hydrogenated.

$T_{\mathrm{c}}$ as a function of the $\mathrm{H}$ content. The second $T_{\mathrm{c}}$ observed is found to be $50-60{ }^{\circ} \mathrm{C}$ lower than the first $T_{\mathrm{c}}$ for all samples. Figure 5 shows the enthalpy change at $T_{\mathrm{m}}$ plotted against the $\mathrm{H}$ molar fraction, which was almost constant independent of the $\mathrm{H}$ content.

The DSC data could then be analyzed from a thermodynamic point of view. ${ }^{18}$ The probabilities for finding a pair of the $\mathrm{H}$ and $\mathrm{H}$ chains $(\mathrm{H}-\mathrm{H}), \mathrm{D}$ and $\mathrm{D}(\mathrm{D}-\mathrm{D})$ chains or $\mathrm{H}$ and $\mathrm{D}(\mathrm{H}-\mathrm{D})$ chains can be expressed approximately as $X^{2},(1-X)^{2}$ or $2 X(1-X)$, respectively, where the $X$ is the molar fraction of the $\mathrm{H}$ species. If we assume the coexistence of the $\mathrm{H}$ and $\mathrm{D}$ chain stems randomly in the common crystallite, then the changes in the enthalpy $(\Delta H)$ and entropy $(\Delta S)$ at the melting point are expressed by the following equations, respectively:

$$
\begin{aligned}
& \Delta H=X^{2} \Delta H_{\mathrm{hh}}+(1-X)^{2} \Delta H_{\mathrm{dd}}+2 X(1-X) \Delta H_{\mathrm{hd}} \\
& \Delta S=X^{2} \Delta S_{\mathrm{hh}}+(1-X)^{2} \Delta S_{\mathrm{dd}}+2 X(1-X) \Delta S_{\mathrm{hd}}
\end{aligned}
$$

where $\Delta H_{\mathrm{hh}}, \Delta H_{\mathrm{dd}}$ and $\Delta H_{\mathrm{hd}}$ represent the enthalpy changes related to the systems that are constructed by the $\mathrm{H}-\mathrm{H}, \mathrm{D}-\mathrm{D}$ and $\mathrm{H}-\mathrm{D}$ pairs, respectively. At the equilibrium melting point $T_{\mathrm{m}}^{\mathrm{o}}, \Delta H$ and $\Delta S$ are obtained:

$$
T_{\mathrm{m}}^{\mathrm{o}}=\frac{X^{2} \Delta H_{\mathrm{hh}}+(1-X)^{2} \Delta H_{\mathrm{dd}}+2 X(1-X) \Delta H_{\mathrm{hd}}}{X^{2} \Delta S_{\mathrm{hh}}+(1-X)^{2} \Delta S_{\mathrm{dd}}+2 X(1-X) \Delta S_{\mathrm{hd}}}
$$

The interatomic interactions between the $\mathrm{H}$ and $\mathrm{D}$ species are essentially the same as these interactions originate from the Coulombic interactions between electrons and protons. The assumption of $\Delta H_{\mathrm{hh}}=\Delta H_{\mathrm{hd}}=\Delta H_{\mathrm{dd}}$ in Equation (3) is reasonable. If the entropy terms $\Delta S_{\mathrm{hh}}, \Delta S_{\mathrm{hd}}$ and $\Delta S_{\mathrm{dd}}$ are also assumed to be the same, then the $T_{\mathrm{m}}^{\mathrm{o}}$ given in Equation (3) no longer depends on the H/D. However, the entropy terms cannot be assumed to be identical, as is clearly shown by the difference in the observed melting points between the blend samples. In general, the entropy term $\Delta S$ is influenced by many contributions, such as the conformational difference between the crystalline and melted states (conformational entropy; $\Delta S_{\text {conf }}$ ), the vibrational entropy; $\Delta S_{\text {vib }}$ and the statistically irregular packing of the different species (packing entropy; $\Delta S_{\text {pack }}$ ). Among these sources, the conformational entropy $\Delta S_{\text {conf }}$ is reasonably assumed to be nearly identical between the $\mathrm{H}$ and $\mathrm{D}$ chains as the conformational change is mainly due to a change in the skeletal torsional angles, which are essentially identical between these two species. The $\mathrm{CD}_{2}$ and $\mathrm{CH}_{2}$ units 


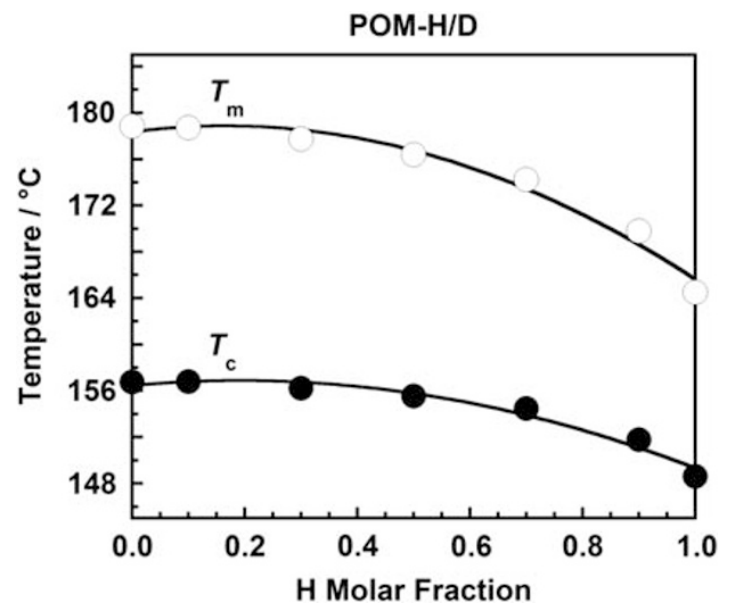

Figure 3 The melting temperature $\left(T_{\mathrm{m}}\right)$ and crystallization temperature $\left(T_{\mathrm{c}}\right)$ as a function of the hydrogenated (H) molar fraction of the polyoxymethylene-H/deuterated blend samples, observed at heating and cooling rates of $2^{\circ} \mathrm{C} \mathrm{min}^{-1}$.

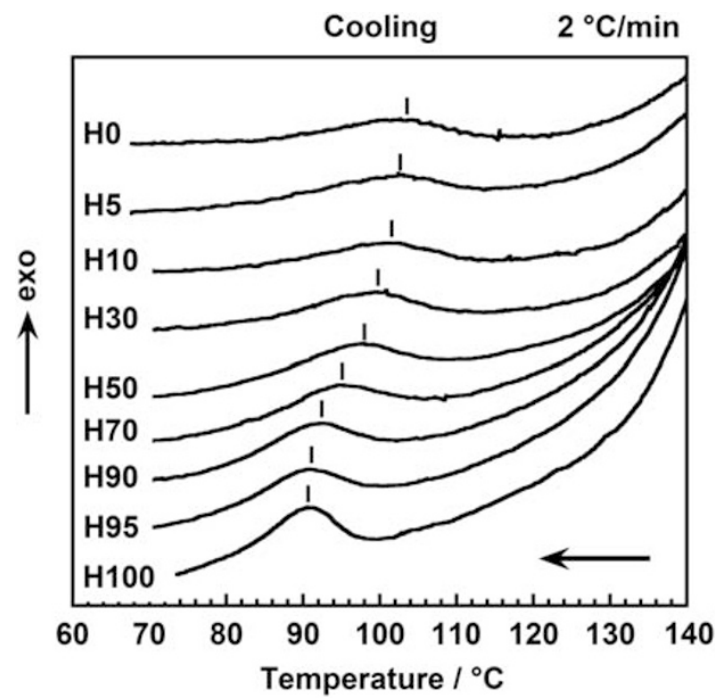

Figure 4 Differential scanning calorimeter thermograms of a series of polyoxymethylene-hydrogenated $(H) /$ deuterated $(D)$ blend samples with various $H / D$ contents during the cooling process, with the second crystallization temperature $\left(T_{\mathrm{c} 2}\right)$ shown.

have different masses, and the vibrational frequencies differ as well. Therefore, the vibrational entropy $\Delta S_{\text {vib }}$ is appreciably different between these two species. The main reason for the different entropy terms in Equations (2) and (3) $\left(\Delta S_{\mathrm{hh}} \neq \Delta S_{\mathrm{hd}} \neq \Delta S_{\mathrm{dd}}\right)$ is the difference in the vibrational entropy term. As a result, the entropy change due to the random arrangement of the $\mathrm{H}$ and $\mathrm{D}$ chain stems in the crystal lattice $\left(\Delta S_{\text {pack }}\right)$, which is expressed in Equation (2), differs depending on the H/D content. Another noteworthy point in the discussion of Equation (3) pertains to the degree of crystallinity of the samples. The crystallinity was estimated using the X-ray diffraction data and was found to be nearly identical for all of the samples used here. Therefore, the effect of crystallinity can conveniently be canceled out in the treatment of Equation (3). Now, utilizing the relation of $\Delta S_{\mathrm{hd}}=\Delta H_{\mathrm{hd}}$ / $T_{\text {mhd }}^{\mathrm{o}}$ and so on, Equation (3) can be rewritten as below, in which

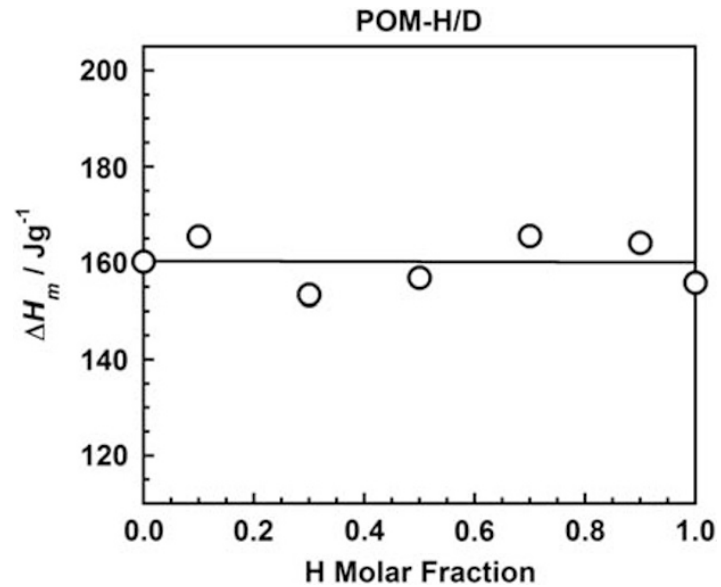

Figure 5 Observed enthalpy of melt for a series of polyoxymethylenehydrogenated $(\mathrm{H})$ /deuterated $(\mathrm{D})$ blend samples with various $\mathrm{H} / \mathrm{D}$ contents.

$T_{\mathrm{mhh}}^{\mathrm{o}}, T_{\mathrm{mdd}}^{\mathrm{o}}$ and $T_{\mathrm{mhd}}^{\mathrm{o}}$ are the equilibrium melting temperatures of the pure $\mathrm{H}, \mathrm{D}$ and $\mathrm{H} / \mathrm{D}$ blend samples, respectively.

$$
T_{\mathrm{m}}^{\mathrm{o}}=\frac{1}{X^{2} / T_{\mathrm{mhh}}^{\mathrm{o}}+(1-X)^{2} / T_{\mathrm{mdd}}^{\mathrm{o}}+2 X(1-X) T_{\mathrm{mhd}}^{\mathrm{o}}}
$$

or

$$
1 / T_{\mathrm{m}}^{\mathrm{o}}=X^{2} / T_{\mathrm{mhh}}^{\mathrm{o}}+(1-X)^{2} / T_{\mathrm{mdd}}^{\mathrm{o}}+2 X(1-X) / T_{\mathrm{mhd}}^{\mathrm{o}}
$$

Equation (5) shows the relationship between the equilibrium melting point $T_{\mathrm{m}}^{\mathrm{o}}$ and the molar content $X$. As can be noticed in the equation, the observed $T_{\mathrm{m}}$ cannot be used, but rather the so-called equilibrium melting temperature $T_{\mathrm{m}}^{\mathrm{o}}$ must be applied for all of the samples of the various $\mathrm{H} / \mathrm{D}$ blend contents.

The equilibrium melting temperature $T_{\mathrm{m}}^{\mathrm{o}}$ may be defined as the melting temperature of an ideal crystal of an infinitely large size. The Gibbs-Thomson equation, derived from thermodynamical relations, is given below. ${ }^{37}$

$$
T_{\mathrm{m}}(l)=T_{\mathrm{m}}^{\mathrm{o}}\left[1-\frac{2}{\Delta H_{\mathrm{f}}}\left(\frac{\sigma_{\mathrm{e}}}{l}+\frac{\sigma}{b}+\frac{\sigma}{a}\right)\right]
$$

where $\sigma$ and $\sigma_{\mathrm{e}}$ are the free energies of the lateral $(a \times l$ and $b \times l)$ and end surfaces $(a \times b)$ of the lamella, with the dimensions $a \times b \times l$, and $\Delta H_{\mathrm{f}}$ is the heat of fusion per unit volume of the repeating unit $\left(53 \mathrm{cal} \mathrm{cm}^{-3}\right) .{ }^{38}$ In general, $a$ and $b$ are much larger than $l$, especially for the FCC structure. Thus, Equation (6) is approximated by

$$
T_{m}(l) \cong T_{\mathrm{m}}^{\mathrm{o}}-\frac{C}{l}
$$

where $C=2 \sigma_{\mathrm{e}} T_{\mathrm{m}}^{\mathrm{o}} / \Delta H_{\mathrm{f}}$. The plot of $T_{\mathrm{m}}(l)$ vs $l^{-1}$ yields the $T_{\mathrm{m}}^{\mathrm{o}}$ from the intercept of the expected straight line. Of course, the $\sigma_{\mathrm{e}}$ may also be estimated from the slope of this straight line, but this value might be affected by the value $\Delta H_{\mathrm{f}}$. As our purpose is only to estimate, we will skip the discussion of ambiguity in $\sigma_{\mathrm{e}}$.

Figure 6 shows the Gibbs-Thomson plot constructed for POM$\mathrm{H} 100$ and POM-H0 as examples. The crystalline thickness $l$ was evaluated by estimating the FWHM $(\beta)$ for the 009 X-ray reflection, as described in the experimental section. The evaluated values of $\sigma_{\mathrm{e}}(\mathrm{s})$ were $5.04 \pm 0.12 \times 10^{-10} \mathrm{~J} \mathrm{~m}^{-2}$ for POM-H100 and $8.97 \pm 0.09 \times 10^{-10}$ $\mathrm{J} \mathrm{m}^{-2}$ for POM-H0. Similarly, the $\sigma_{\mathrm{e}}(\mathrm{s})$ were estimated as $5.03 \pm 0.22$ $\times 10^{-10}, \quad 5.59 \pm 0.1 \times 10^{-10}, \quad 6.47 \pm 0.07 \times 10^{-10}, \quad 8.06 \pm 0.05 \times 10^{-10}$ and $8.98 \pm 0.22 \times 10^{-10} \mathrm{~J} \mathrm{~m}^{-2}$ for H90, H70, H50, H30 and H10, 


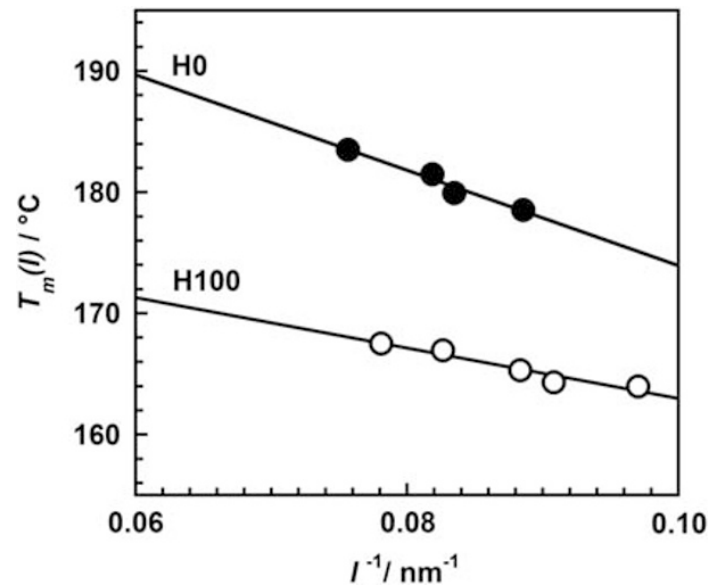

Figure 6 Gibbs-Thomson plots to evaluate the equilibrium melting temperature, where only two cases, polyoxymethylene (POM)-HO and POM$\mathrm{H} 100$, are shown as examples.

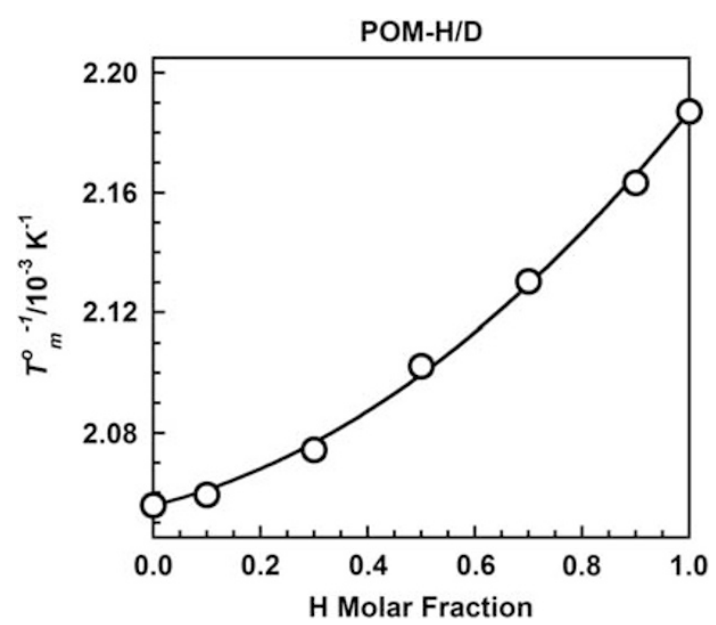

Figure 7 Dependence of an equilibrium melting temperature $T_{\mathrm{m}}^{\circ}$ on the hydrogenated molar fraction. The solid line is the curve that was calculated using Equation (5) in the text, where an adjustable parameter $T_{\text {mhd }}^{\circ}=208^{\circ} \mathrm{C}$ was assumed.

respectively. As the value of $\sigma_{\mathrm{e}}$ is related to the work of forming a chain fold, the lowering of energy caused by the blending of the $\mathrm{H}$ and $\mathrm{D}$ species makes it possible for the $\mathrm{H}$ and $\mathrm{D}$ chains to be crystallized in the common crystalline lattice.

The $T_{\mathrm{m}}^{\mathrm{o}}$ of pure POM-H100 was estimated to be $184.1 \pm 1.0^{\circ} \mathrm{C}$ and that of pure POM-D (POMH0) was estimated at $213.3 \pm 0.5^{\circ} \mathrm{C}$. Correspondingly, the $T_{\mathrm{m}}^{\mathrm{o}}(\mathrm{s})$ were estimated as $189.1 \pm 2.1,196.3 \pm$ 2.0, $202.5 \pm 0.5,208.9 \pm 0.3$ and $212.4 \pm 1.2^{\circ} \mathrm{C}$ for H90, H70, H50, $\mathrm{H} 30$ and $\mathrm{H} 10$, respectively. The $\mathrm{T}_{\mathrm{m}}^{\mathrm{o}}$ of POM-H100 (Delrin 550, homopolymer) was reported to be $210^{\circ} \mathrm{C}$ by Carter et al.,$^{39} 206^{\circ} \mathrm{C}$ by Majer ${ }^{40}$ and $199.7^{\circ} \mathrm{C}$ by Wissbrun. ${ }^{41}$ The $T_{\mathrm{m}}^{\mathrm{o}}$ estimated in the present study is lower than that in these reports because a copolymer with ethylene oxide content of $2.2 \mathrm{wt} \%$ was used as the pure POM-H sample (Duracon M90). The $T_{\mathrm{m}}^{\mathrm{o}}$ (s) obtained for all of the samples were used to check the reasonableness of Equation (5). The solid line shown in Figure 7 is the fitted curve, created by assuming that the adjustable parameter $T_{\mathrm{mhd}}^{\mathrm{o}}=208^{\circ} \mathrm{C}$. The $T_{\mathrm{m}}^{\mathrm{o}}$ changes continuously, indicating the perfect cocrystallization of the $\mathrm{D}$ and $\mathrm{H}$ types of POM species.

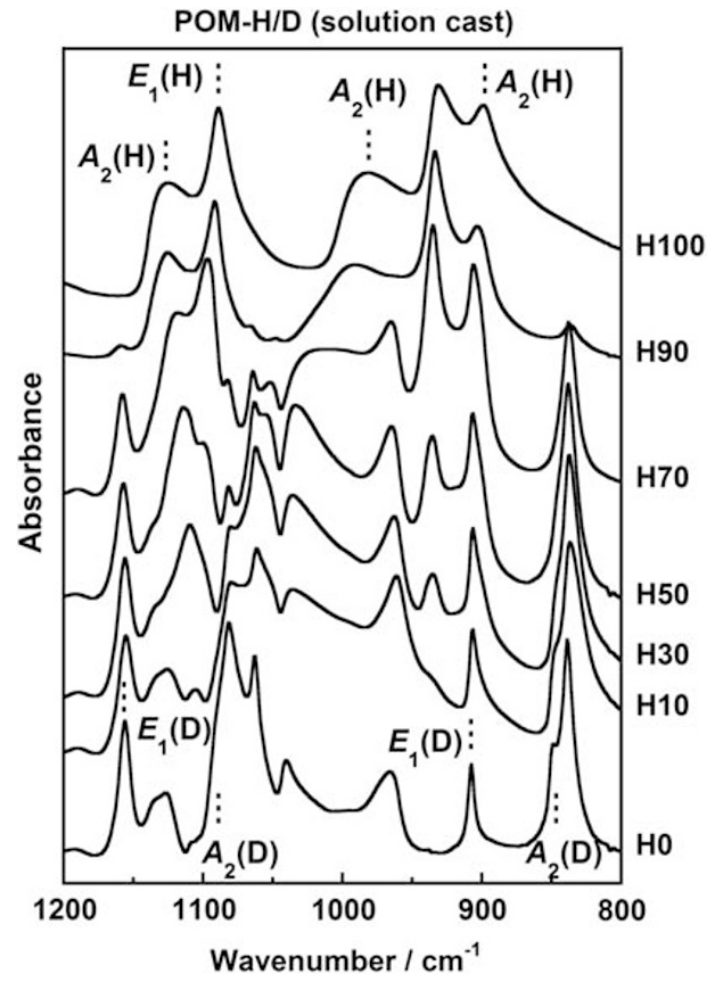

Figure 8 Fourier transform infrared spectra of solution-cast polyoxymethylene-hydrogenated $(H) /$ deuterated $(D)$ blend samples with various $H / D$ compositions, measured at room temperature.

Cocrystallization phenomena as viewed by vibrational spectroscopy To confirm the cocrystallization phenomena as revealed by the DSC data, the IR spectra were measured for a series of the blend samples. It should again be pointed out that the IR spectra of POM are strongly dependent on the morphology of the sample. At the same time, the coexistence of the $\mathrm{D}$ and $\mathrm{H}$ chain stems in the crystalline lamellae may also affect sensitively the spectral profiles. As the purpose of the present study is to confirm the coexistence of the $\mathrm{H}$ and $\mathrm{D}$ chains in the crystalline region, it is important to use a common morphology adjustment for all of the samples. The single crystals grown from the dilute solutions or the FCC were prepared as having the typical morphology. For the IR spectral measurement, the dilute solution of POM in 1,1,1,3,3,3-hexafluoro-2-propanol solution $(\sim 1 \mathrm{wt} \%)$ was cast onto a $\mathrm{KBr}$ single-crystal plate. The evaporation rate of the solvent was reduced by reducing the crystallization temperature. Figure 8 shows the series of IR spectra that were observed for the samples with different $\mathrm{H} / \mathrm{D}$ compositions. Judging from the spectral profile of POM-H0 and POM-H100, the samples commonly have the FCC morphology. As another morphological characteristic, the IR spectra were measured for the melt-cooled samples as shown in Figure 9.

As mentioned previously, the drastic shift in the parallel bands depending on the morphology can be interpreted by assuming that there are long-range interactions among the transition dipoles. ${ }^{27,28}$ Vibrations of the CO bonds induce the transition dipoles, and the interactions between these dipoles affect the vibrational frequency in a feedback manner. The vibrational frequency shift is expressed by the following equation:

$$
\Delta v=(1 / v)\left(\partial \mu / \partial Q_{k}\right)^{2} \sum_{j}\left(1 / R_{j}^{3}\right)
$$




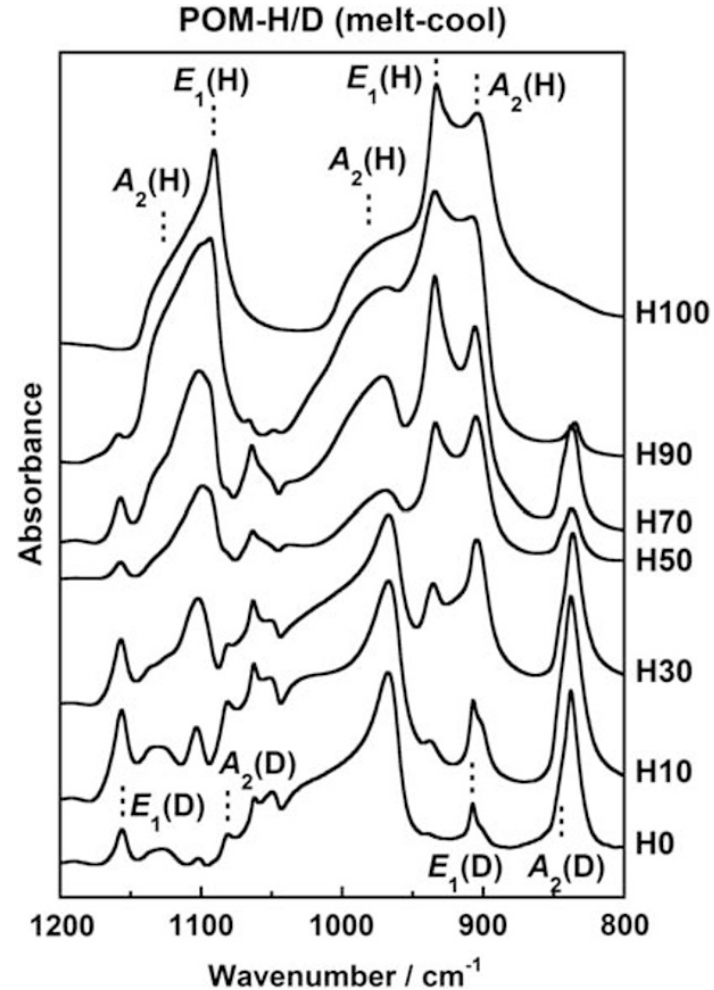

Figure 9 Fourier transform infrared spectra of melt-cooled polyoxymethylenehydrogenated $(H) /$ deuterated (D) blend samples with various $H / D$ compositions, measured at room temperature.

where $v$ is the vibrational frequency, $\left(\partial \mu / \partial Q_{k}\right)$ is the transition dipole moment and $R_{j}$ is the lateral distance of the $j$ th chain from the central chain. ${ }^{28}$ The total sum of dipole-dipole interactions over the crystalline region is determined by the geometry or the morphology of the crystal phase. Vibrational coupling between the transition dipoles can, in principle, occur for all of the substances, but the magnitude of the frequency shift is dependent on the strength of the dipole-dipole interactions. ${ }^{28}$ The $\mathrm{C}-\mathrm{O}$ bonds of POM give very large dipoles, which result in the remarkably large frequency shifts observed.

Now, let us analyze the blend sample of POM-D and -H species. We assume that the normal POM chains or the hydrogenous POM chains are homogeneously blended with the fully D POM chains in the common crystal lattice. The $\mathrm{H}$ and $\mathrm{D}$ chain species show different vibrational frequencies because of differences in the atomic mass. By changing the H/D blend ratio, the IR spectral profile will be changed, as illustrated in Figure 10a. At the same time, however, we must consider the effect of vibrational couplings between the transition dipoles. As the $\mathrm{CD}_{2} \mathrm{O}$ units vibrate at much lower frequencies than the $\mathrm{CH}_{2} \mathrm{O}$ units, the vibrational couplings between the $\mathrm{CH}_{2} \mathrm{O}$ units $\left(\mathrm{CD}_{2} \mathrm{O}\right.$ units) are blocked when the $\mathrm{CD}_{2} \mathrm{O}\left(\mathrm{CH}_{2} \mathrm{O}\right)$ groups penetrate into the aggregated space of the $\mathrm{CH}_{2} \mathrm{O}\left(\mathrm{CD}_{2} \mathrm{O}\right)$ groups, where the couplings between the $\mathrm{CH}_{2} \mathrm{O}$ and $\mathrm{CD}_{2} \mathrm{O}$ units are almost negligible. As a result, the total sum of the dipole-dipole interactions expressed in Equation (8) is modified, and the parallel bands of the $\mathrm{CH}_{2} \mathrm{O}\left(\mathrm{CD}_{2} \mathrm{O}\right)$ units or the $A_{2}$ bands will show remarkable changes in the vibrational frequencies compared with the original values, as shown in Figure $10 \mathrm{~b}{ }^{28}$ The $E_{1}$ modes with transition dipoles that are perpendicular to the chain axis are doubly degenerated and are not affected by the dipole-dipole interactions. ${ }^{28}$ In this manner, the blending of the $\mathrm{H}$ and D-POM chains in the crystal lattice affects the IR spectra of POM

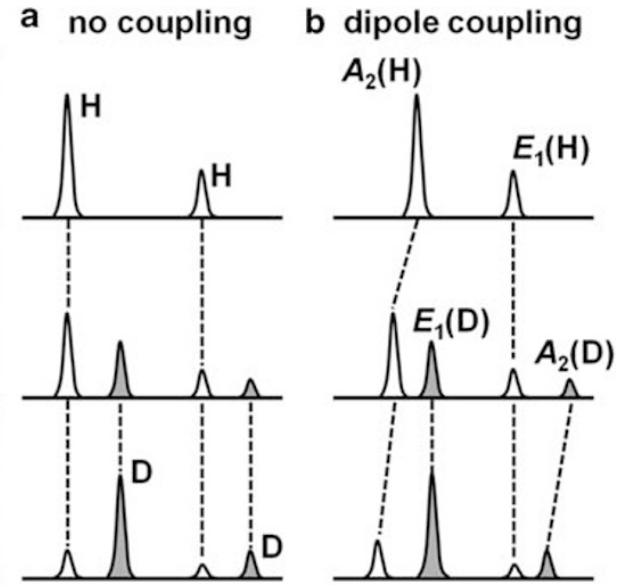

Figure 10 Schematic illustration of the relationship between the molecular aggregation state and the infrared spectral profile. (a) No dipole-dipole interactions between the same types of chain stems exist, and the relative band intensities change almost in proportion to the hydrogenated $(H) /$ deuterated (D) content. (b) The position and relative intensity of the $A_{2}$ bands, with transition dipoles parallel to the chain axis, are shifted depending on the spatial distribution of the $H$ and $D$ chain stems in the crystal lattice. The doubly degenerated $E_{1}$ mode bands do not show any frequency shift as the transition dipoles are perpendicular to the chain axis. $^{28}$ Some of the bands detected in Figures 8 and 9 are assigned to the $A_{2}$ and $E_{1}$ bands of the $\mathrm{H}$ and $\mathrm{D}$ species.

more markedly than is expected from the simple change in the relative intensity of the vibrational bands, as illustrated in Figure 10a. As indicated in Figures 8 and 9, the complicated spectral changes that are observed for the blend samples may also be interpreted in this manner as long as the $\mathrm{D}$ and $\mathrm{H}$ chain stems are mixed together randomly in the common lattice. As the domain sizes of the $\mathrm{H}$ (and D) species surrounded by the $\mathrm{D}$ (and $\mathrm{H}$ ) species in the crystalline region may distribute statistically randomly, the summation of the dipole-dipole interactions changes in a complicated manner, making it difficult to reasonably simulate the observed spectral changes. The interpretation of these remarkable changes in the spectral profiles is now being processed on the basis of the equation of dipole-dipole coupling.

The cocrystallization can also be confirmed by observing the simultaneous appearance of the crystalline bands of the $\mathrm{H}$ and $\mathrm{D}$ chains in the crystallization process from the melt. For example, Figure 11 shows the temperature dependence of the IP spectra that were measured for the POM-H50 blend sample during the cooling process from the melting point at a cooling rate of $2{ }^{\circ} \mathrm{C} \mathrm{min}-1$. As plotted in Figure $11 \mathrm{~b}$, the $\mathrm{H}$ band $\left(1237 \mathrm{~cm}^{-1}\right)$ and the $\mathrm{D}$ band $\left(1158 \mathrm{~cm}^{-1}\right)$ were found to begin simultaneously appearing at around $159^{\circ} \mathrm{C}$. This clearly indicates that the $\mathrm{D}$ and $\mathrm{H}$ chains approach the frontier plane of the growing lamella almost simultaneously and are trapped together as crystalline stems, as illustrated in Figure 12.

\section{CONCLUSION}

The continuous changes observed in the DSC thermogram and IR spectra for a series of POM-H/D blend samples have been reasonably interpreted by assuming the random distribution of the $\mathrm{H}$ and $\mathrm{D}$ chain stems in the common crystalline lattice or with a concept of cocrystallization between the $\mathrm{H}$ and $\mathrm{D}$ chains. The continuous shift of the equilibrium melting temperature was reasonably analyzed by the random distribution of the $\mathrm{H}$ and $\mathrm{D}$ chain stems. The simultaneous appearance of the IR crystalline bands of the $\mathrm{H}$ and $\mathrm{D}$ species also 
a
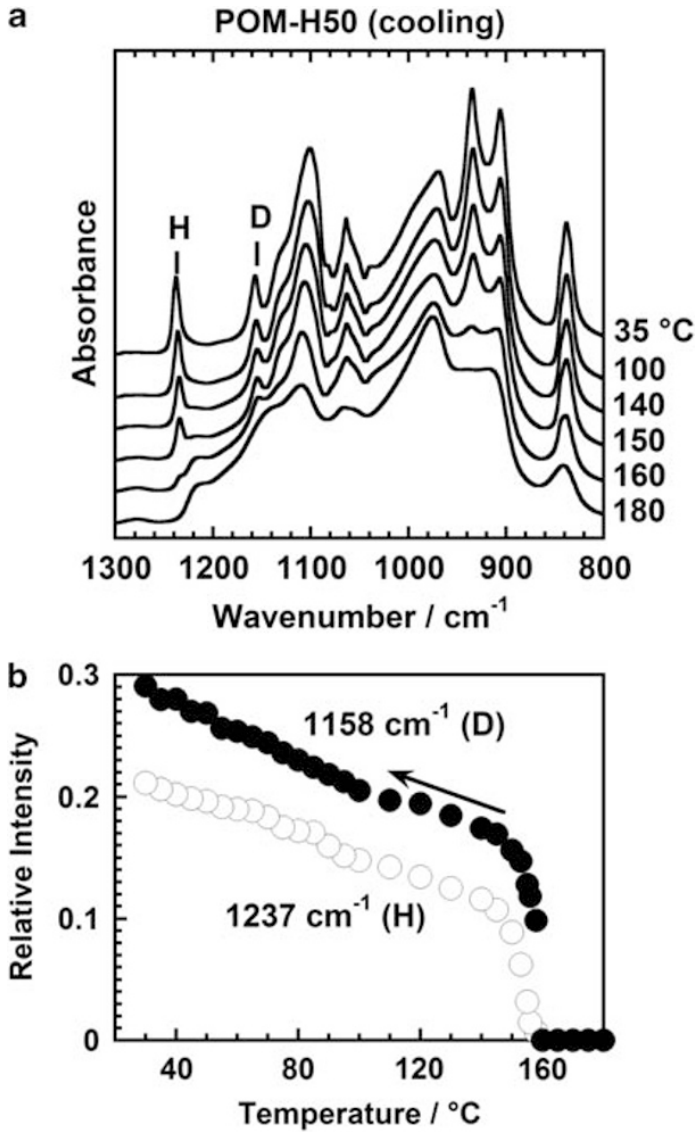

Figure 11 (a) Temperature dependent Fourier transform infrared spectra and (b) the integrated intensity of the hydrogenated $(\mathrm{H})$ bands $\left(1238 \mathrm{~cm}^{-1}\right)$ and deuterated bands $\left(1158 \mathrm{~cm}^{-1}\right)$ observed for the polyoxymethylene-H5O blend sample during the cooling process from the melting point.

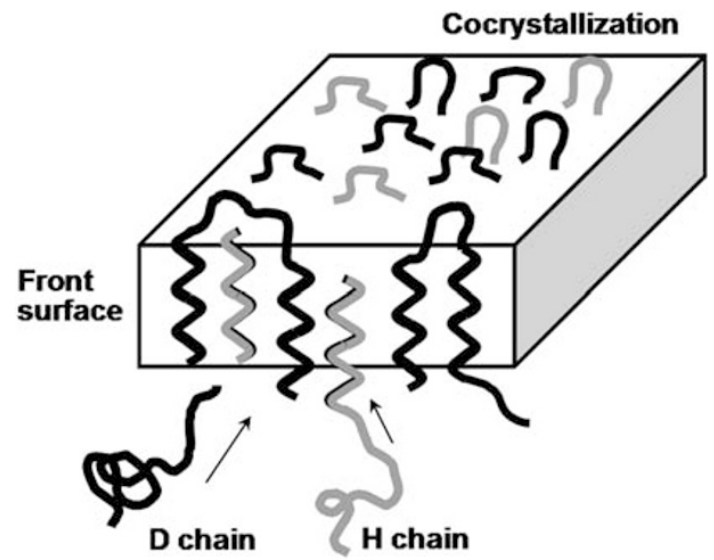

Figure 12 Schematic illustration of the cocrystallization process of the polyoxymethylene (POM)-deuterated (D) and POM-hydrogenated (H) chains, where the $\mathrm{D}$ and $\mathrm{H}$ chains simultaneously approach the front surface of the lamella.

supports this concept. It should be noticed, however, that the thermal and FTIR data reported in the present paper are only a portion of the experimental evidence that supports the concept of cocrystallization of the POM-D and -H species. The wide-angle X-ray diffraction profiles, which are not given in the present paper, have been found to be essentially the same among all of the $\mathrm{D} / \mathrm{H}$ samples used here, telling us that the crystal structures are the same for all of the samples independent of the $\mathrm{D} / \mathrm{H}$ content. From all of these experimental data, it is not unreasonable to conclude that cocrystallization occurs between the $\mathrm{D}$ and $\mathrm{H}$ species of POM when they are cooled from the melting point. As for the IR spectral data, it may in principle be possible to deduce the spatial distribution of the $\mathrm{H}$ and $\mathrm{D}$ chains in the crystallization process on the basis of the evolution of the IR spectral profile. The details of this analysis will be reported elsewhere.

\section{ACKNOWLEDGEMENTS}

This work was supported by the MEXT 'Collaboration with Local Communities' Project (2005-2009).

1 Tasumi, M. \& Krimm, S. Crystal vibrations of polyethylene. J. Chem. Phys. 46, 755-766 (1967).

2 Stehling, F. C., Ergos, E. \& Mandelkern, L. Phase separation in n-hexatriacontane-nhexatriacontane- $\mathrm{d}_{74}$ and polyethylene-poly(ethylene- $\left.\mathrm{d}_{4}\right)$ systems. Macromolecules 4, 672-677 (1971)

3 Krimm, S. \& Ching, J. H. C. Infrared spectra of polyethylene-poly(ethylene-d 4 ) mixedcrystal systems. Macromolecules 5, 209-211 (1972).

4 Shelten, J., Wignall, G. D. \& Ballard, D. G. H. Chain conformation in molten polyethylene by low angle neutron scattering. Polymer 15, 682-685 (1974).

5 Shelten, J., Ballard, D. G. H., Wignall, G. D., Longman, G. W. \& Schmatz, W. Smallangle neutron scattering studies of molten and crystalline polyethylene. Polymer 17, 751-757 (1976)

6 Shelten, J., Ballard, D. G. H., Wignall, G. D., Longman, G. W. \& Schmatz, W. Neutron small-angle scattering by mixtures of $\mathrm{H}$ and $\mathrm{D}$-tagged molecules of polystyrene and polyethylene. Colloid Polym. Sci. 252, 749-752 (1974).

7 Shelten, J., Wignall, G. D., Ballard, D. G. H. \& Longman, G. W. Small-angle neutron scattering studies of molecular clustering in mixtures of polyethylene and deuterated polyethylene. Polymer 18, 1111-1120 (1977).

8 Yoon, D. Y. \& Flory, P. J. Small-angle X-ray and neutron scattering by polymethylene, polyoxyethylene, and polystyrene chains. Macromolecules 9, 294-299 (1976).

9 Tashiro, K., Stein, R. S. \& Hsu, S. L. Cocrystallization and phase segregation of polyethylene blends. 1 . Thermal and vibrational spectroscopic study by utilizing the deuteration technique. Macromolecules 25, 1801-1808 (1992).

10 Tashiro, K., Satkowski, M. M., Stein, R. S., Li, Y., Chu, B. \& Hsu, S. L. Cocrystallization and phase segregation of polyethylene blends. 2. Synchrotron-sourced X-ray scattering and small-angle light scattering study of the blends between the $\mathrm{D}$ and $\mathrm{H}$ species. Macromolecules 25, 1809-1815 (1992).

11 Tashiro, K., Izuchi, M., Kobayashi, M. \& Stein, R. S. Cocrystallization and phase segregation of polyethylene blends between the $\mathrm{D}$ and $\mathrm{H}$ species. 3. Blend content dependence of the crystallization behavior. Macromolecules 27, 1221-1227 (1994).

12 Tashiro, K., Izuchi, M., Kobayashi, M. \& Stein, R. S. Cocrystallization and phase segregation of polyethylene blends between the $\mathrm{D}$ and $\mathrm{H}$ species. 4 . The crystallization behavior as viewed from the infrared spectral changes. Macromolecules 27, 1228-1233 (1994).

13 Tashiro, K., Izuchi, M., Kobayashi, M. \& Stein, R. S. Cocrystallization and phase segregation of polyethylene blends between the $\mathrm{D}$ and $\mathrm{H}$ species. 5. Structural studies of the blends as viewed from different levels of unit cell to spherulite. Macromolecules 27, 1234-1239 (1994).

14 Alamo, R. G., Londono, J. D., Mandelkern, L., Stehling, F. C. \& Wignall, G. D. Phase behavior of blends of linear and branched polyethylenes in the molten and solid states by small-angle neutron scattering. Macromolecules 27, 411-417 (1994).

15 Wignall, G. D., Londono, J. D., Alamo, R. G., Lin, J. S., Galante, M. J. \& Mandelkern, L. Morphology of blends of linear and long-chain-branched polyethylenes in the solid state: a study by SANS, SAXS, and DSC. Macromolecules 28, 3156-3167 (1995).

16 Tashiro, K., Izuchi, M., Kaneuchi, F., Jin, C., Kobayashi, M. \& Stein, R. S. Cocrystallization and phase segregation of polyethylene blends between the $\mathrm{D}$ and $\mathrm{H}$ species. 6 . Time-resolved FTIR measurements for studying the crystallization kinetics of the blends under isothermal conditions. Macromolecules 27, 1240-1244 (1994).

17 Tashiro, K., Imanishi, K., Izuchi, M., Kobayashi, M., Itoh, Y., Imai, M., Yamaguchi, Y., Ohashi, M. \& Stein, R. S. Cocrystallization and phase segregation of polyethylene blends between the $\mathrm{D}$ and $\mathrm{H}$ species. 8. Small-angle neutron scattering study of the molten state and the structural relationship of chains between the melt and the crystalline state. Macromolecules 28, 8484-8490 (1995).

18 Sasaki, S., Tashiro, K., Gose, N., Imanishi, K., Izuchi, M., Kobayashi, M., Imai, M., Ohashi, M., Yasuo, Y. \& Ohayama, K. Spatial distribution of chain stems and chain folding mode in polyethylene lamellae as revealed by coupled information of DSC, FTIR, SANS, and WANS. Polym. J. 31, 677-686 (1999).

19 Tashiro, K. \& Gose, N. Diffusion and aggregation of hydrogeneous and deuterated polyethylene chains at their interfacial boundary as studied by time- and space-resolved FTIR microscopic measurements. Polymer 42, 8987-8998 (2001). 
20 Reddy, K. R., Tashiro, K., Sakurai, T. \& Yamaguchi, N. Cocrystallization phenomenon between the $\mathrm{H}$ and $\mathrm{D}$ Species of isotactic polypropylene blends as revealed by thermal and infrared spectroscopic analyses for a series of $\mathrm{D} / \mathrm{H}$ blend samples. Macromolecules 41, 9807-9813 (2008).

21 Reddy, K. R., Tashiro, K., Sakurai, T. \& Yamaguchi, N. Isotope effect on the isothermal crystallization behavior of isotactic polypropylene blends between the deuterated and hydrogenated species. Macromolecules 42, 1672-1678 (2009).

22 Reddy, K. R., Tashiro, K., Sakurai, T., Yamaguchi, N., Sasaki, S., Masunaga, M. \& Takata, M. Isothermal crystallization behavior of isotactic polypropylene H/D blends as viewed from time-resolved FTIR and synchrotron SAXS/WAXD measurements. Macromolecules 42, 4191-4199 (2009).

23 Tadokoro, H., Yasumoto, S., Murahashi, S. \& Nitta, I. Molecular configuration of polyoxymethylene. J. Polym. Sci. 44, 266-269 (1960).

24 Tashiro, K., Hanesaka, M., Ohhara, M., Ozeki, T., Kitano, T., Nishu, T., Kurihara, K., Tamada, T., Kuroki, R., Fujiwara, S., Tanaka, I. \& Niimura, N. Structural refinement and extraction of hydrogen atomic positions in polyoxymethylene crystal based on the first successful measurements of 2-dimensional high-energy synchrotron X-ray diffraction and wide-angle neutron diffraction patterns of hydrogenated and deuterated species. Polymer J. 39, 1253-1273 (2007).

25 Kobayashi, M. \& Matsumoto, Y. Morphology dependent anomalous frequency shifts of infrared parallel bands of polymer crystals: spectral changes induced by isotopic dilution in H-D cocrystals of poly(oxymethylene) as evidence of a transition dipolar coupling mechanism. Macromolecules 28, 1580-1585 (1995).

26 Shimomura, M., Iguchi, M. \& Kobayashi, M. Vibrational spectroscopic study on trigonal polyoxymethylene and polyoxymethylene- $d_{2}$ crystals. Polymer 29, 351-357 (1988).

27 Kobayashi, M., Morishita, H. \& Shimomura, M. Pressure-induced phase transition of poly(oxymethylene) from the trigonal to the orthorhombic phase: effect of morphological structure. Macromolecules 22, 3726-3730 (1989).

28 Kobayashi, M. \& Sakashita, M. Morphology dependent anomalous frequency shifts of infrared absorption bands of polymer crystals: interpretation in terms of transition dipole-dipole coupling theory. J. Chem. Phys. 96, 748-760 (1992).
29 Bassett, D. C., Dammont, F. R. \& Salovey, R. On the morphology of polymer crystals. Polymer 5, 579-588 (1964).

30 Iguchi, M. Growth of needle-like crystals of polyoxymethylene during polymerization. Br. Polym. J. 5, 195-198 (1973).

31 Iguchi, M. \& Murase, I. Growth of needle-like polyoxymethylene crystals. J. Cryst. Growth 24/25, 596-599 (1974).

32 Hama, H. \& Tashiro, K. Structural changes in non-isothermal crystallization process of melt-cooled polyoxymethylene. [I] Detection of infrared bands characteristic of folded and extended chain crystal morphologies and extraction of a lamellar stacking model. Polymer 44, 3107-3116 (2003).

33 Hama, H. \& Tashiro, K. Structural changes in non-isothermal crystallization process of melt-cooled polyoxymethylene. [II] Evolution of lamellar stacking structure derived from SAXS and WAXS data analysis. Polymer 44, 2159-2168 (2003).

34 Hama, H. \& Tashiro, K. Structural changes in isothermal crystallization process of polyoxymethylene investigated by time-resolved FTIR, SAXS and WAXS measurements. Polymer 44, 6973-6988 (2003).

35 Okamura, S., Hayashi, K. \& Nakamura, Y. Radiation induced polymerization of trioxane. Isotope and Radiation 3, 416 (1960).

36 Hayashi, K., Nishii, M. \& Okamura, S. Structure of polymers formed by radiationinduced solid phase polymerization of cyclic monomers. J. Polym. Sci. C4, 839-847 (1964).

37 Wunderlich, B Macromolecular Physics, Vol. 3: Crystal Melting 1-127 (Academic Press, New York, 1980).

38 Limbert, F. J. \& Baer, E. Kinetics of nucleation and growth of spherulites in homopolymers. J. Polymer Sci. Pt. A 1, 3317-3331 (1993).

39 Carter, D. R. \& Baer, E. Lamellar crystallization and melting of polyoxymethylene. J. Appl. Phys. 37, 4060-4065 (1966).

40 Majer, J. Rate of growth of poly(oxymethylene) spherulites. Kunststoffe 52, 535-536 (1962).

41 Wissbrun, K. F. The equilibrium melting point of polyoxymethylene. J. Polym. Sci Part A-2 4, 827-829 (1966) 